\title{
Numerical Estimation of Ohmic Loss of High Power Wideband Diplexer for ECCD System*)
}

\author{
Kohei ATSUMI, Tomoki YAMAGUCHI, Koji NAGASHIMA, Mikio SAIGUSA, \\ Masafumi FUKUNARI ${ }^{1)}$, Yasuhisa ODA ${ }^{1)}$ and Keishi SAKAMOTO ${ }^{1)}$ \\ College of Engineering, Ibaraki University, Hitachi, Ibaraki 316-8511, Japan \\ 1) Japan Atomic Energy Agency, Naka, Ibaraki 311-0193, Japan
}

(Received 28 November 2012 / Accepted 2 May 2013)

\begin{abstract}
A diplexer for high power millimeter wave has been developed as a fast switching device in ECCD system for improving a stabilizing efficiency of neoclassical tearing modes. The switching operation of diplexer was confirmed with the FDTD code developed in our laboratory and low power tests using a mock-up diplexer. The cooling design of half mirror will be a key issue of this development, so that the Ohmic loss of a miter bend and a half mirror of the ring resonator was estimated by the numerical simulations.
\end{abstract}

(c) 2013 The Japan Society of Plasma Science and Nuclear Fusion Research

Keywords: diplexer, NTM, ECCD, fast switching device, half mirror

DOI: $10.1585 /$ pfr.8.2405077

\section{Introduction}

Neo-classical tearing modes (NTMs) with rotate at $5-10 \mathrm{kHz}$ deteriorate energy confinement of fusion plasma in tokamaks [1]. Electron cyclotron current drive (ECCD) using high power millimeter wave could suppress NTMs [2]. Local current drive into O-point of a magnetic island of NTM is useful for NTM stabilization. Especially, a fast directional switch makes the duty of ECCD system to $100 \%$ by switching beam direction for tracking the rotating O-point of a magnetic island of NTM [3]. For a frequency tunable ECCD system, the new wide band diplexer as a fast switching device was proposed [4]. The switching operation of a wideband diplexer was confirmed by numerical simulations and by low power tests $[5,6]$.

In this paper, the preliminary results of numerical simulations and low power tests of diplexer with new metal half mirrors are reported. At first, the principle of diplexer is explained in Sec. 2. Low power tests of a designed diplexer are described in Sec. 3. The numerical simulation results of a electromagnetic field and the preliminary results of numerical simulations of Ohmic loss are described in Sec. 4. Finally, the study is summarized in Sec. 5.

\section{Principle of Diplexer}

The proposed diplexer consists of a circular corrugated waveguides, two miter bends and two half mirrors as shown in Fig. 1. A propagating mode is $\mathrm{HE}_{11}$ mode of which transmission loss is very low. The incident wave is divided into the reflected wave and the transmitted wave by the half mirror 1 as shown in Fig. 2. The transmitted

author's e-mail: saigusa@mx.ibaraki.ac.jp

*) This article is based on the presentation at the 22nd International Toki Conference (ITC22).

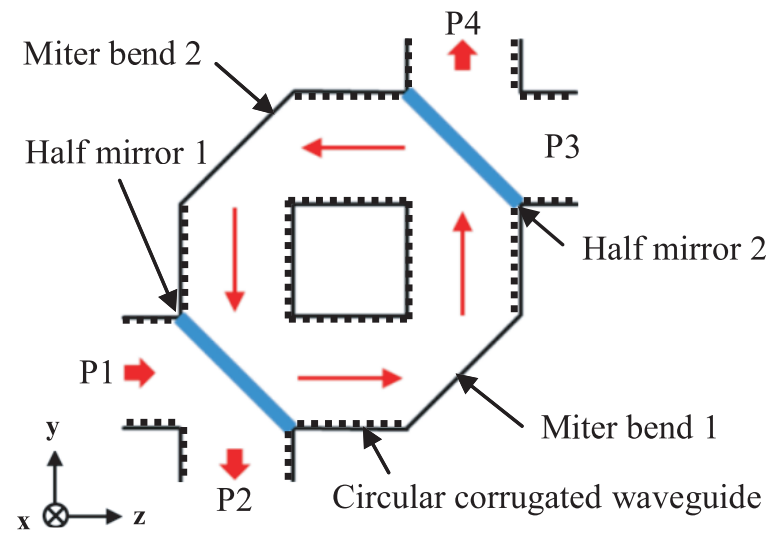

Fig. 1 Principle of fast switching using a diplexer with shifting a frequency.

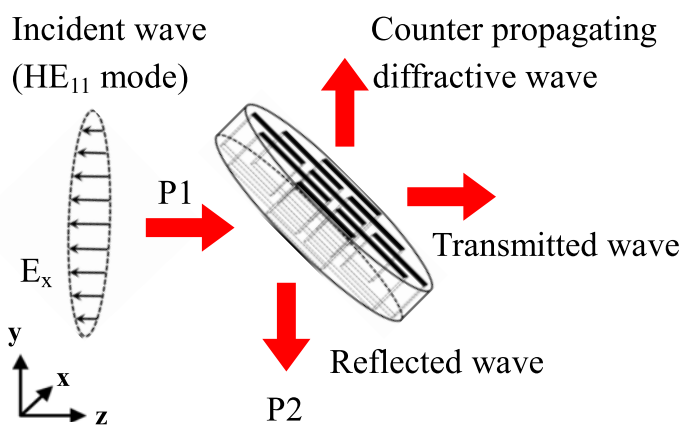

Fig. 2 Schematic view of a slotted metal half mirror.

electromagnetic wave is entered in a ring resonator. If it is a resonance frequency of a ring resonator, $\mathrm{rf}$ power are accumulated in a resonant ring and the output power from P4 increases. On the other hand, in the case of no reso- 
nance, most power goes to $\mathrm{P} 2$. So, the output power can be switched by shifting a frequency.

When $P$ and $a$ are the input power of P1 and the power reflection coefficient of the half mirror 1 and 2, the output powers of P2 and P4 can be obtained as follows, where $\theta$ is the delayed phase in the round trip length of the ring resonator.

$$
\begin{aligned}
& P_{4}=\frac{(1-a)^{2}}{1+a^{2}-2 a \cos \theta} \\
& P_{2}=1-P_{4}=1-\frac{(1-a)^{2}}{1+a^{2}-2 a \cos \theta} .
\end{aligned}
$$

\section{Low Power Tests}

The designed diplexer using slotted metal half mirror has been tested with low power at wide frequency band from $169.5 \mathrm{GHz}$ to $170.5 \mathrm{GHz}$. The diameter of a circular corrugated waveguide is $63.5 \mathrm{~mm}$, and the length of a resonant ring is $650 \mathrm{~mm}$. In these low power tests, two kinds of short slotted metal half mirrors were used as shown in Fig. 3. Because, heat is conducted only one dimension in a long slotted half mirror (central slot length $=92 \mathrm{~mm}$ ), while the heat can be expanded two dimensions in a short slotted half mirror [7]. The mirror thickness was designed to be $1.7 \mathrm{~mm}$ by numerical simulations for a suitable power reflection coefficient in both of slotted metal disk and diamond one [4].

The reflection coefficients of half mirrors should be

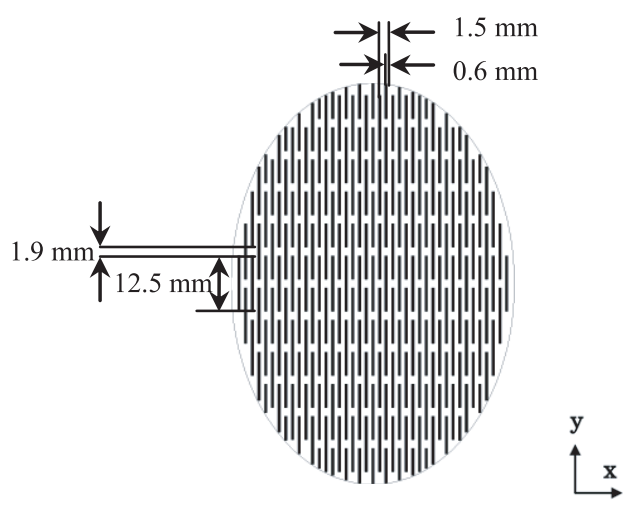

(a) Short slotted mirror A.

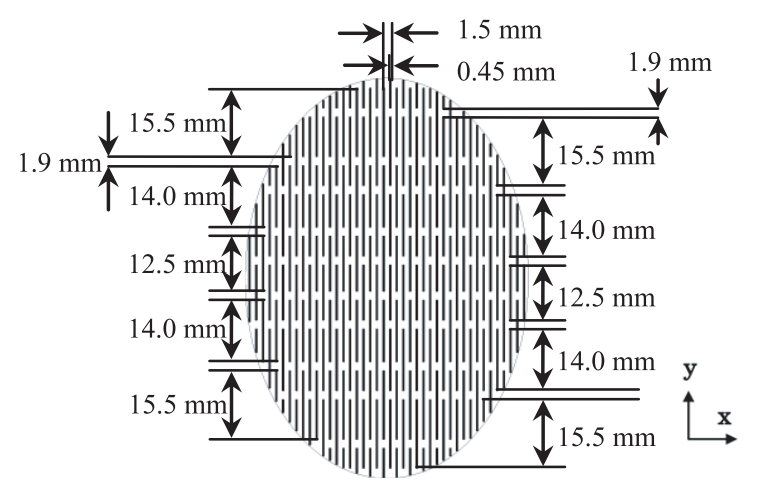

(b) Short slotted mirror B.

Fig. 3 Slot size of used short slotted metal half mirror. designed considering the gyrotron frequency band, the resonant length and loss of a ring resonator. Short slotted mirror $\mathrm{A}$ is the periodical slot structure in $\mathrm{x}$-direction and $\mathrm{y}$-direction. The slot period in $\mathrm{x}$-direction and the slot width are $1.5 \mathrm{~mm}$ and $0.6 \mathrm{~mm}$, respectively. The slot period in x-direction: $1.5 \mathrm{~mm}$ is shorter than wavelength at $170 \mathrm{GHz}(\fallingdotseq 1.76 \mathrm{~mm})$. However, the slot period in $y-$ direction: $14.4 \mathrm{~mm}$ is much wider than a wave length, so that high order diffraction must be excited in y-direction. On the other hand, short slotted mirror B is the nonperiodical slot structure in y-direction in order to control high order diffractions. The slot lengths in y-direction are $12.5 \mathrm{~mm}, 14 \mathrm{~mm}$, and $15 \mathrm{~mm}$ as it goes outside from inside. The slot width in $\mathrm{x}$-direction: $0.45 \mathrm{~mm}$ is smaller than that of short slotted mirror A in order to raise a reflection coefficient.

The reflected and transmitted powers from a half mirror installed in a cross corrugated waveguide were measured with a waveguide antenna scanning on 2-D stage [7]. The measured reflection and transmission coefficients were about $60 \%$ and $40 \%$ in the mirror A, and they were about $75 \%$ and $25 \%$ in the mirror $\mathrm{B}$, respectively. The radiation patterns of reflection and transmission look like the pattern of $\mathrm{HE}_{11}$ mode. The counter propagating diffractive waves defined in Fig. 2 are generated by the high order diffraction. The radiation patterns in Fig. 4 indicate that the high order diffractions were suppressed by the non-periodic slot structure of the mirror B.

The frequency dependence of the diplexer using mirror $\mathrm{B}$ at the frequency band of $170 \mathrm{GHz}$ was measured.

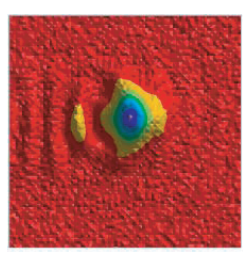

(a) mirror $\mathrm{A}$

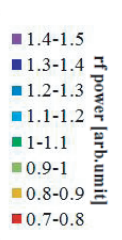

$0.8-0.9 \equiv$

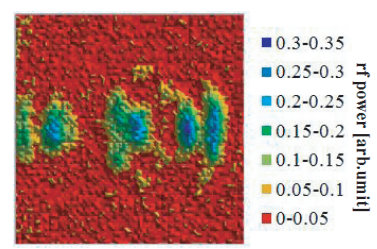

(b) mirror B
Fig. 4 Radiation patterns of counter propagating diffractive waves with short slotted mirror A and short slotted mirror $\mathrm{B}$. The measuring area is $150 \mathrm{~mm} \times 150 \mathrm{~mm}$ in both cases.

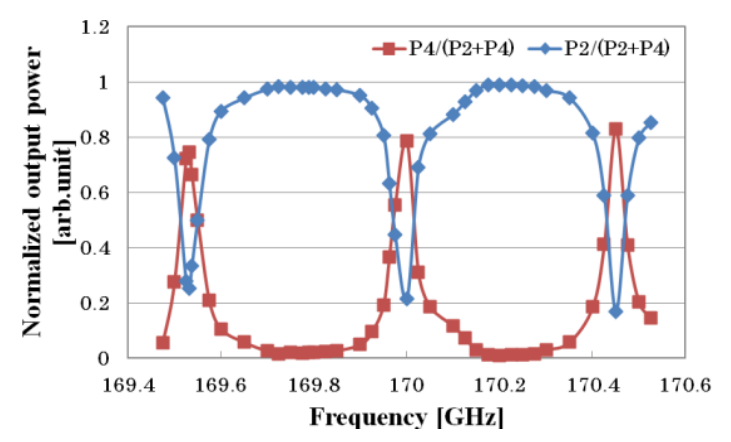

Fig. 5 Frequency dependence of P2 and P4 output powers using mirror B in low power test. 
Table 1 The parameter of numerical simulations.

\begin{tabular}{|c|c|}
\hline Cell size $[\mathrm{mm}]$ & $0.15 \times 0.15 \times 0.15$ \\
\hline Total cell count $(x \times y \times z)$ & $151 \times 450 \times 450$ \\
\hline Time step $[\mathrm{s}]$ & $2.86 \times 10^{-13}$ \\
\hline Number of steps & 15000 \\
\hline
\end{tabular}

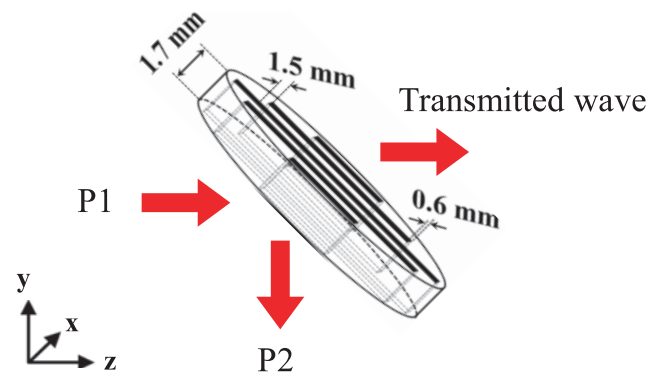

Fig. 6 Slot size of used long slotted metal half mirror.

Switching properties were clearly observed as shown in Fig. 5.

\section{Numerical Simulations}

The electromagnetic field in the diplexer has been simulated with our developed code using FDTD method. The slotted metal plates are used as the half mirrors installed in the ring resonator. Long slotted metal half mirror made of aluminum alloy is assumed in this simulation. The slot period is $1.5 \mathrm{~mm}$ (Air: Metal $=0.6 \mathrm{~mm}: 0.9 \mathrm{~mm}$ ) as show in Fig. 6, which is smaller than a wavelength: $1.76 \mathrm{~mm}$ at an input frequency of $170 \mathrm{GHz}$. The diameter of corrugated waveguides and the one turn length of a ring resonator are assumed to be $21.0 \mathrm{~mm}$, and $135 \mathrm{~mm}$, respectively, due to the memory limitation (12 GB). The used cell size is smaller than one tenth of a wavelength of an input frequency.

The simulation results at the resonant frequency are shown in Fig. 7. It was confirmed that the output power from $\mathrm{P} 4$ increases with decreasing the output power from $\mathrm{P} 2$, at a resonant condition. The power loss of a metal surface can be calculated as the follows, where $H_{\mathrm{t}}$ is a tangential component of magnetic field, $R_{\mathrm{S}}$ is a rf sheet resistance, $\sigma$ is an electrical conductivity, and $\delta$ is a skin depth.

$$
P_{\text {loss }}=\frac{1}{2}\left|H_{\mathrm{t}}\right|^{2} R_{\mathrm{s}}=\frac{1}{2}\left|H_{\mathrm{t}}\right|^{2} \frac{1}{\delta \sigma} .
$$

Since a magnetic field component is not given to a metal surface of miter bend, the magnetic field component of the metal surface is interpolated by adjacent point's data as show in Fig. 8.

The tangential component of a magnetic field $H_{\mathrm{t}}$ is computed by a vector calculation using the magnetic field component of the metal surface as follows.

$$
H_{\mathrm{t}}=\sqrt{\left(\frac{H_{y}^{2}+H_{z}^{2}}{\sqrt{2}}\right)^{2}+H_{x}^{2}} .
$$

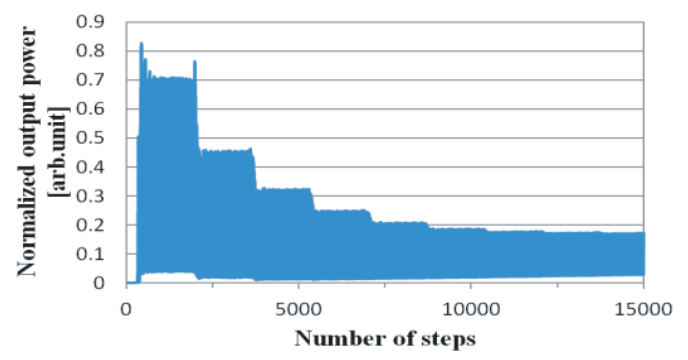

(a) Output from P2

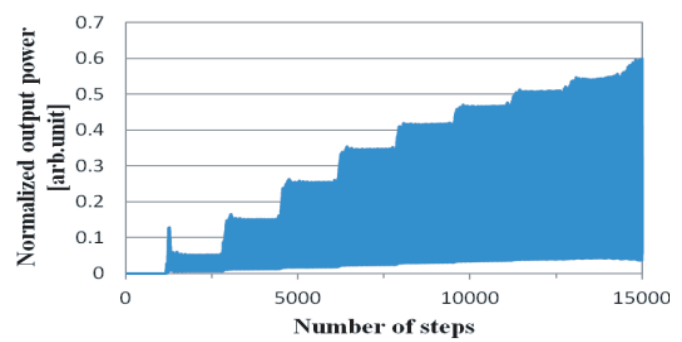

(b) Output from P4

Fig. 7 Time evolution of normalized rf power of diplexer using slotted metal half mirrors simulated by FDTD code at a resonant condition of ring resonator.

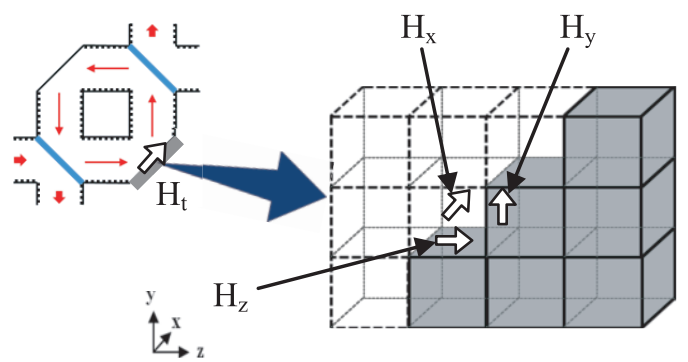

Fig. 8 Schema of the tangential component of a magnetic field $H_{\mathrm{t}}$ using FDTD method.

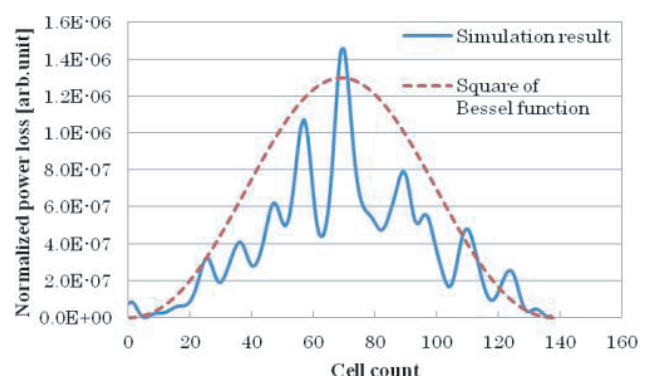

Fig. 9 Profile of Ohmic loss on the reflected surface of the miter bend 1 installed in the ring resonator simulated by FDTD code.

Ohmic loss at a miter bend and a slotted half mirror installed in a ring resonator was simulated. It will become important to design the slotted half mirror considering heat load in high power operation. The heat load was estimated by the numerical simulations of Ohmic loss. Since the power loss in a ring resonator is also calculable by these 


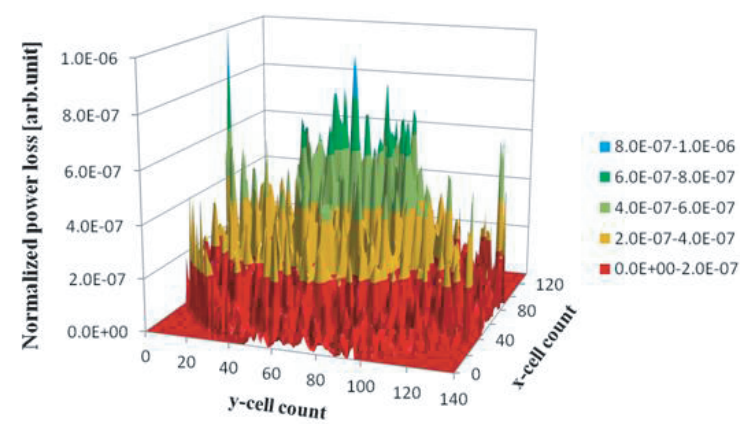

(a) A bird's-eye view of 3D-Ohmic loss profile

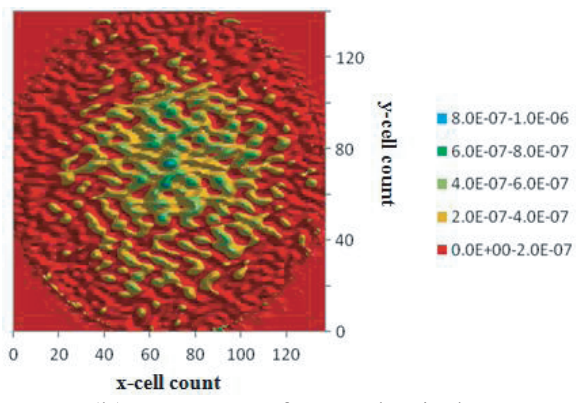

(b) Contour of 2D-Ohmic loss

Fig. 10 Profiles of Ohmic loss on the surface of the miter bend 2 in Fig. 1.

numerical simulations, it can be used also for evaluation of the switching operation.

The results of Ohmic loss at miter bend 1 installed in ring resonator are shown in Fig. 9. The solid line indicates the simulation result, while the dotted line shows the theoretical prediction without higher modes. The difference between both lines suggests the existence of higher modes in an oversized corrugated waveguide ring.

The simulated results of 3D Ohmic loss profile at a miter bend 2 installed in ring resonator are shown in Fig. 10. It was confirmed that there was a peak value of Ohmic loss in the center of miter bend 2 .

The profiles of Ohmic loss on the input-side surface of the half mirror 2 are shown in Fig. 11. There is a peak value of Ohmic loss in the center of slotted half mirror 2 like $\mathrm{HE}_{11}$ mode.

The Ohmic loss on the surfaces of the miter bend 1 and the miter bend 2, the inner surfaces of the half mirror 1 and the half mirror 2 were estimated numerically to be $0.35 \%, 0.26 \%, 0.25 \%$, and $0.18 \%$, respectively. Therefore, the total Ohmic loss in a ring resonator is estimated to be $1.05 \%$.

\section{Summary}

The high power wide band diplexer was designed and fabricated as a fast switching device for ECCD system.

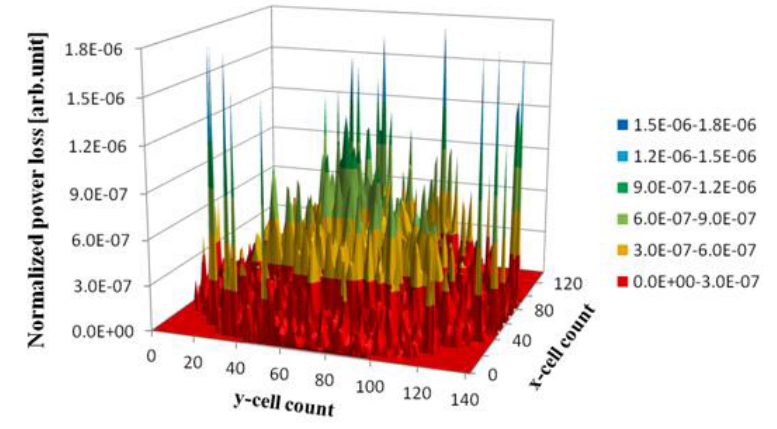

(a) A bird's-eye view of 3D-Ohmic loss profile

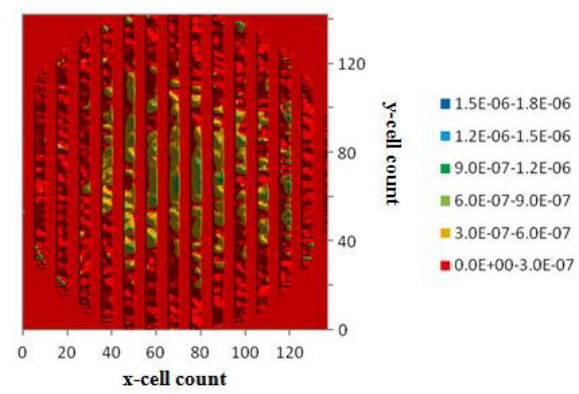

(b) Contour of 2D-Ohmic loss

Fig. 11 3D-Ohmic loss profile on the half mirror 2 surface installed in the ring resonator.

The switching operation was confirmed by numerical simulations and by low power tests. Ohmic loss at a miter bend and metal slotted half mirror installed in a ring resonator was simulated. In the both cases, Ohmic loss was confirmed. There are two ideas for cooling mirrors. One is the thick half mirror made of OFHC with inside cooling channels. The other is the thin half mirror made of Glidcop with edge cooling. The heat conduction and thermal stress analyses of the half mirror are planed in near future.

\section{Acknowledgments}

This work has been supported by the Grants-in-aid for Scientific Research (c) of 22560818.

[1] Z. Chang et al., Phys. Rev. Let. 74, 4663 (1995).

[2] H. Zohm, Phys. Plasmas 4, 3433 (1997).

[3] W. Kasparek et al., Fusion Eng. Des. 84, 1002 (2009).

[4] M. Saigusa et al., Proc. of 13th AMPERE Toulouse (2011) p.285.

[5] K. Atsumi et al., "Research and Development of High Power Wideband Diplexer for ECCD System” Plasma Conference 2011, Kanazawa, 23P099-P (2011).

[6] M. Saigusa et al., Plasma Fusion Res. 7, 2405099 (2012).

[7] M. Saigusa et al., /10.1016/j.fusengdes.2013.02.163, Fusion Eng. Des. (2013) (in press). 